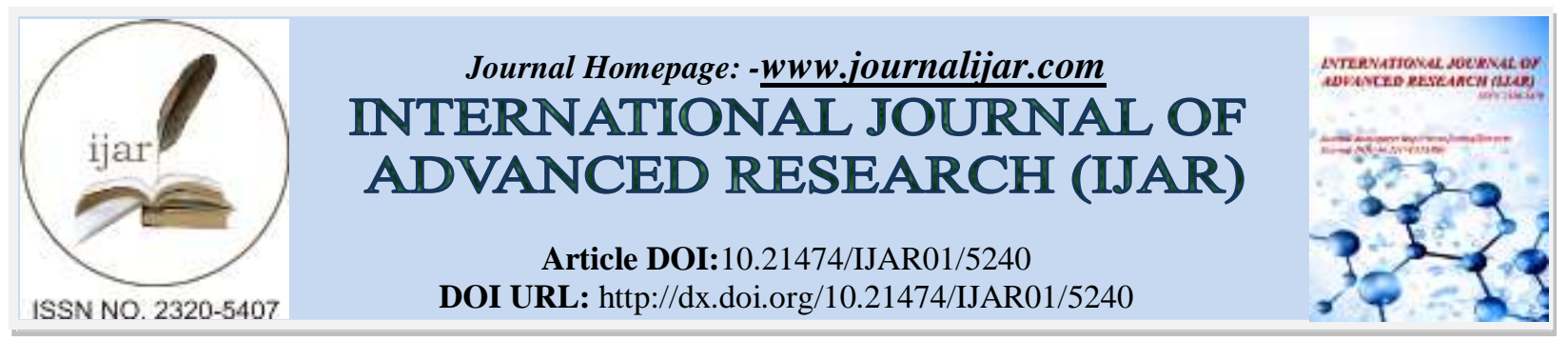

RESEARCH ARTICLE

\title{
SPONTANEOUS PARAVERTEBRAL HEMATOMA: A CASE REPORT.
}

\section{Erguibi, A. Elbakouri, T. Brouzine, R. Boufettal, S.R. Jai And F. Chehab.}

1. Service de Chirurgie viscerale aile 3 au CHU Ibn Rochd, Casablanca, Faculté de médecine et de pharmacie Casablanca, Université Hassan II Casablanca MAROC.

2. Driss ERGUIBI Professeur Assistant chirurgie viscerale.

3. Abdelilah ELBAKOURI Resident en chirurgie viscerale.

4. Taghrid BROUZINE Interne en chirurgie viscerale.

5. Rachid BOUFETTAL Professeur agregé en chirurgie viscerale.

6. Saad Rifki JAI Professeur de l'enseignement superieur en chirurgie viscerale.

7. Farid CHEHAB Professeur de l'enseignement superieur en chirurgie viscerale, chef de service Aile 3 et Doyen de la faculté de Medecine et de Pharmacie de Casablanca MAROC.

\section{Manuscript Info}

Manuscript History

Received: 21 June 2017

Final Accepted: 23 July 2017

Published: August 2017

Key words:-

Hematoma, retroperitoneum

\begin{abstract}
Paravertebral spontaneous hematomas are rare and often reveal an underlying pathology, particularly infectious, tumorous or haematological. The symptomatology is usually acute, but progressive forms are possible.

We report the observation of a 37-year-old woman, a left paravertebral hematoma was discovered as part of the etiological assessment of a recent onset abdominal pain syndrome. Abdominal CT scan revealed a large left paravertebral mass of $10 \mathrm{~cm}$ long axis. A median laparotomy was done. The tumor was soft and movable. A total exeresis was performed. The pathology examination had conclude in a calcified hematoma. The follow-up was 3 years.
\end{abstract}

Copy Right, IJAR, 2017,. All rights reserved.

\section{Introduction:-}

Les hematomes spontanés intra-abdominaux sont rares et révèlent souvent une pathologie sous-jacente. Ils posent des difficultés diagnostiques et thérapeutiques.

Le but de cette mise au point est de préciser la stratégie diagnostique et thérapeutique devant les hematomes spontanés intra-abdominaux.

\section{Observation:-}

Une femme âgée de 37 ans, hospitalisée pour le bilan étiologique d'un hematome para-vertébral spontané. Il ne signale aucun antécédent familial ou personnel évoquant un contexte de maladie hémorragique familiale.

La patiente décrit l'apparition spontanée, un an auparavant, d'une douleur de l'hypochondre gauche, sans notion de traumatisme récent. 
L'examen Clinique est sans particularité hormis une sensibilité à la palpation de l'hypochondre gauche. L'échographie, puis l'examen tomodensitométrique, objectivent une formation kystique latéro-pancréatique gauche de $10 \mathrm{~cm}$ de grand axe (figure 1, 2).

L'exploration chirurgicale trouve un hematoma calcifié paravertébral gauche de $12 \mathrm{~cm}$ de grand axe sans pouvoir identifier son origine (figure 3), une exérèse complète a été réalisée. L'examen anatomopathologique a confirmé le diagnostic. Les suites opératoires étaient simples. Le recul est de 3 ans.

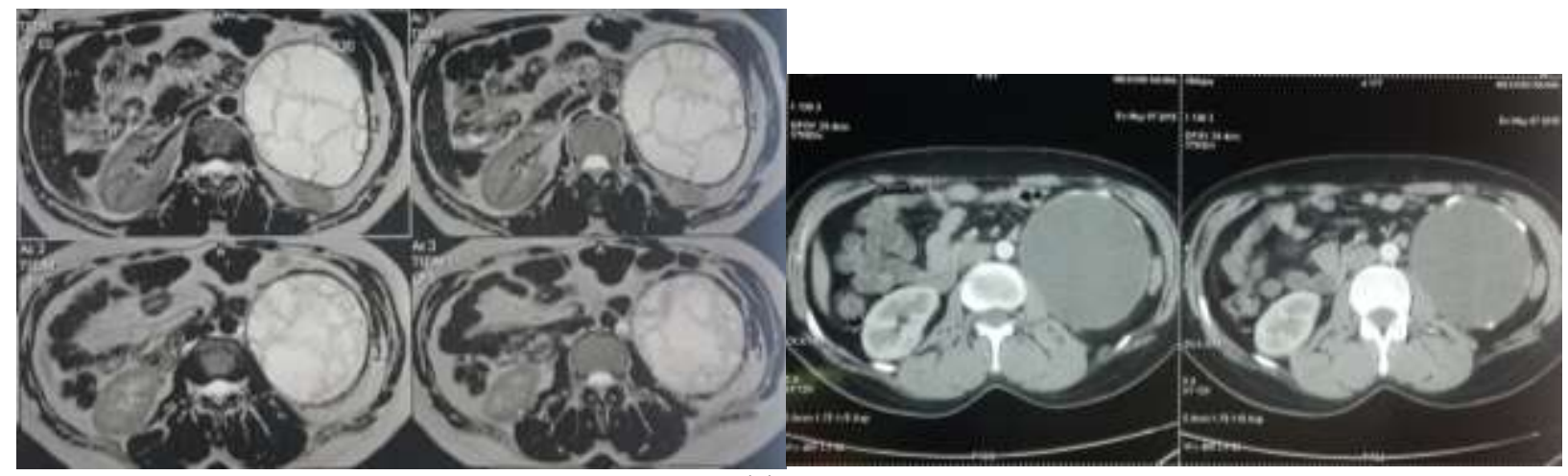

Figure 1 et 2:- Images scanographiques montrant l'hématome paravertébral gauche de $10 \mathrm{~cm}$ de grand axe.

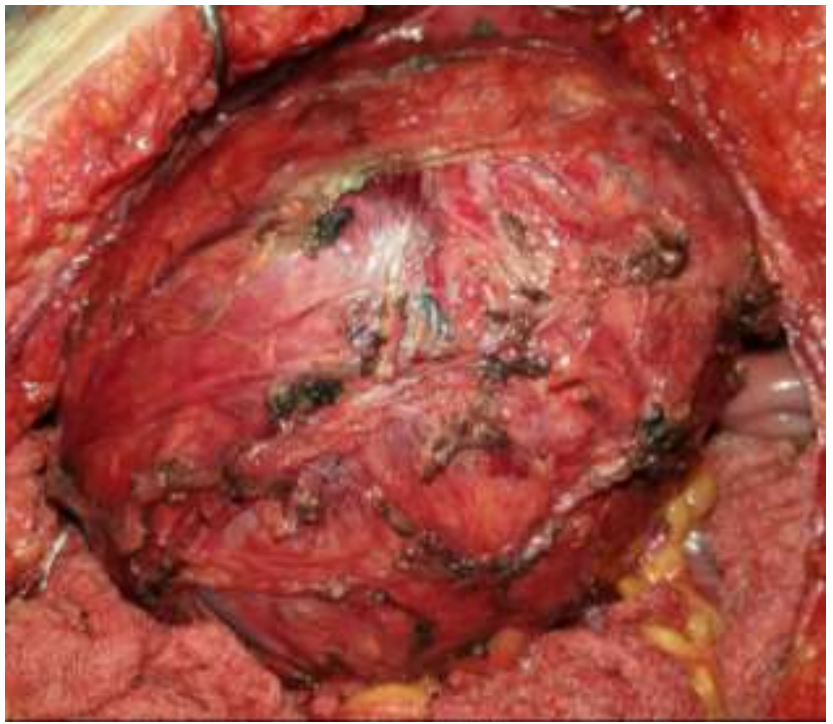

Figure 2:-Image peropératoire montrant l'hématome retropéritonéal.

\section{Discussion:-}

Les hematomes intra-péritonéaux sont des complications fréquentes des polytraumatismes ou des traumatismes abdominaux sévères. Hormis ce contexte particulier, c'est un événement relativement rare qui peut révéler des pathologies infectieuses, tumorales ou pancréatiques. Elle peut être favorisée par la prise d'anti-agrégants plaquettaires ou d'anti-coagulants, mais peut survenir spontanément ou après un traumatisme minime passé inaperçu [1].

Un traitement par anti-coagulation orale au long cours augmente le risque de développer des complications hémorragiques sévères multi-organes, abdominales dans près de la moitié des cas et qui comprennent les saignements intra-luminaux, intra-muraux de l'intestin grêle, les hématomes de la paroi abdominale et les hémorragies rétropéritonéales [1]. 
Chez notre patient l'enquête étiologique s'est avérée négative. Il n'existait aucun signe clinique ou paraclinique en faveur d'une cirrhose. L'absence de syndrome Clinique et biologique infectieux ou encore de voyage à l'étranger rendait peu probable l'origine infectieuse. Une maladie systémique ou une hémopathie ont a priori été éliminées [2]. Cliniquement, l'hématome est souvent asymptomatique mais le risque de rupture spontanée est élevé et la difficulté du diagnostic constitue une des causes de mortalité importante.

L'échographie abdominale, en général facile d'accès, aide au diagnostic en mettant en évidence le plus souvent une masse abdominale, néanmoins de localization parfois difficile à déterminer en fonction des conditions d'examens [3].

Le scanner abdominal sans injection identifie l'hématome spontané, le localisant précisément. L'aspect tomodensitométrique de l'hématome depend également de l'intervalle de temps entre sa constitution et son diagnostic : précocement et dans les deux premières semaines la collection apparaît spontanément hyperdense (4070 Unités Hounsfield), pour devenir par la suite isodense (20-30 UH) entre la deuxième et quatrième semaine, parfois avec la formation de cloisons, et évolue plus tardivement vers la collection hypodense, mimant une formation kystique [3].

Dans notre cas nous avions été consultés pour donner un avis sur la prise en charge d'une tumeur de la queue du pancréas, mais l'atypie de la presentation clinique et la relecture du scanner ont permis de redresser le diagnostic et de retenir celui d'hématome. La conduite à tenir dépend de l'importance de l'hématome, de son retentissement hémodynamique et secondairement de l'étiologie.

La prise en charge d'un hématome du mésentère sera fonction de la tolerance clinique du patient. Des constantes hémodynamiques stables, un diagnostic bien établi permettront la mise en place d'un traitement medical comprenant une surveillance Clinique étroite, des examens biologiques répétés, une surveillance échographiquesi contributive ou scanographique. Dans ce cas, le risque vital ne devra jamais être sous-estimé. Des signes cliniques d'hémorragie sévère, une irritation péritonéale généralisée, une complication surajoutée justifieront un traitement chirurgical d'urgence. L'absence de diminution progressive, en volume et en densités pontanée, de l'hématome nécessitera une exploration chirurgicale afin d'y recherché une lesion tumorale sous-jacente [3, 4].

\section{Conclusion:-}

L'hématome spontané intra-péritonéal est une affection rare, de diagnostic souvent difficile entament sur la prise en charge therapeutique.

Le traitement conservateur semble être une alternative sous réserve de certaines conditions.

\section{References:-}

1. J.-C. Chakarian, C. Belizna, F. Héron, F. Hervé. H. Maillochaud, I. Marie, H. Lévesque. Hématome spontané de la rate :traumatisme et/ou médicament ? Presse Med $2005 ; 34: 1717-18$.

2. Mounia EL ALAOUI, Damien OLIVIÉ, Yves GANDON, Jean-François BRETAGNE. Hématome spontané péri-pancréatique associé à une sténose du tronc cœliaque. Difficultés diagnostiques et modalités thérapeutiques. Gastroenterol Clin Biol2005;29:1169-1171.

3. P. De Brito, M.-A. Gomez, M. Besson, B. Scotto, D. Alison. Hématome mésentérique : rare complication d'un traitement anticoagulant oral au long cours. Annales de chirurgie 131 (2006) 529-532.

4. D Launay, U Michon-Pasture, Y Bournbar, F Dubrulle, J Bouroz-Joly, E Hachulla, L Lemaitre, B Devulder. Hematome perirenal spontané bilateral :une complication rare de la periaterite noueuse. Rev Med Interne 1998 ; $19: 666-9$. 